\title{
Frequency doubling illusion VEPs and automated perimetry in multiple sclerosis
}

\author{
Rasa Ruseckaite · Teddy Maddess · \\ Gytis Danta $\cdot$ Andrew Charles James
}

Received: 29 January 2006/ Accepted: 5 June 2006/Published online: 12 August 2006

(C) Springer Science+Business Media B.V. 2006

\begin{abstract}
We examined frequency doubling (FD) illusion based automated perimetry (FDT) and dichoptic FD multifocal visual evoked potentials (FDmfVEPs) in Normal and multiple sclerosis (MS) subjects. Contrast thresholds were determined at 17 visual field locations using an FDT perimeter. The stimuli presented to each location were $0.25 \mathrm{cpd}$ gratings presented with rapid $(25 \mathrm{~Hz})$ counterphase flicker and thus displayed the spatial FD illusion. Dichoptic mfVEPs were recorded by concurrently stimulating eight regions/eye with FD stimuli presented at 95\% contrast. Recordings were obtained from 27 Normal subjects, 26 MS patients who had experienced Optic Neuritis $\left(\mathrm{MS}_{\mathrm{ON}}\right)$ and $24 \mathrm{MS}$ patients without a history of $\mathrm{ON}\left(\mathrm{MS}_{\mathrm{NON}}\right)$. The FDT thresholds showed enhanced contrast sensitivity for $\mathrm{MS}_{\mathrm{ON}}$ patients $(P<0.0001)$ but not for $\mathrm{MS}_{\mathrm{NON}}$ patients. Response amplitudes for the central four regions of the mfVEP stimulus were reduced in both patient groups $(P<0.005)$. A classification model based upon the FDT thresholds performed at a specificity of $96 \%$ for a sensitivity of $97 \%$ in $\mathrm{MS}_{\mathrm{ON}}$ patients, but the accuracy (simultaneously largest sensitivities and
\end{abstract}

R. Ruseckaite $(\bowtie) \cdot$ T. Maddess · G. Danta

A. C. James

Visual Sciences Group, Research School of Biological

Sciences, ANU, Canberra, ACT 2000, Australia

e-mail: Rasa.Ruseckaite@anu.edu.au specificities) was poor ( $60 \%)$ in $\mathrm{MS}_{\mathrm{NON}}$ patients. Discriminant models based on the FDT thresholds and FDmfVEPs were able to diagnose more that $90 \% \mathrm{MS}_{\mathrm{ON}}$ patients but performed poorly for $\mathrm{MS}_{\mathrm{NON}}$ patients.

Keywords Multiple sclerosis · Optic neuritis · Frequency doubling illusion $\cdot$ ROCs

\section{Introduction}

The spatial frequency doubling (FD) illusion occurs when low-spatial frequency sinusoidal gratings are presented either with rapid counterphase flicker [1-3], or a rapid onset [4, 5]. Under those conditions the gratings appear to have twice their actual spatial frequency. Contrast thresholds for seeing the FD illusion were found to be highly diagnostic for glaucoma [6-10], leading to the development of the frequency doubling technology (FDT) perimeter [11, 12], which tests the contrast threshold for FD stimuli at many visual field locations.

The FDT perimeter is based upon the idea that low-spatial frequencies combined with high temporal frequencies preferentially stimulate retinal ganglion cells of the magnocellular pathway (M-cells), which are primarily involved in the detection of motion and rapid luminance changes. FD contrast thresholds show very low test-retest 
variability in glaucoma $[8,13]$. This has been suggested to be related to the ability of spatial FD stimuli to directly test the contrast gain control system of M-cells [7, 9]: a defective, and so abnormally fluctuating, retinal gain control system possibly being the source of the poor reproducibility obtained with other automated perimeters [14-16]. Hence measuring the source of the variance, rather than attempting to characterize a fluctuating threshold, is a more tractable process.

Large test-retest variability has also been noted for achromatic automated perimetry in optic neuritis (ON) patients [17]. Two recent studies have reported that the contrast threshold based FDT perimeter was useful in ON $[18,19]$. This is perhaps not unexpected as early atrophy of the CNS in multiple sclerosis (MS) has recently been shown to be a combination of the effects of demyelination, axonal loss, and apoptosis of neurons [review 20]. Thus a reliable test of damage to retinal ganglion cells might be of value in MS. Our recent study using temporally sparse multifocal visual evoked potentials (mfVEPs) [21] indicated that transient onset stimuli are correlated with the degenerative phase of MS that is associated with axon loss [20, 22, 23].

Visual evoked potentials (VEPs) have long been used to aid in the diagnosis of MS and potentially related conditions [24-27]. Multifocal VEPs, in which multiple regions of the visual field are tested concurrently, have detected localized scotomas and response delays in ON [28]. Some of us [10, 29] have shown that suprathreshold FD stimuli can also provide highly accurate diagnosis of glaucoma when employed in a multifocal pattern electroretinogram. Thus, several lines of evidence suggest that differential assessment of the visual field using either threshold or suprathreshold FD stimuli may be of value in MS. This prompted us to investigate MS comparing the diagnostic capacity of the FDT perimeter and a dichoptic mfVEP method that used suprathreshold FD stimuli, the latter being a modification of our PERG method for glaucoma [10, 29]. Dichoptic measurement permits sensitive comparison between eyes given that the response from both eyes is obtained simultaneously through the same recording electronics.
In the present study we investigate MS patients with a history of $\mathrm{ON}\left(\mathrm{MS}_{\mathrm{ON}}\right)$ and MS patients with no history of $\mathrm{ON}\left(\mathrm{MS}_{\mathrm{NON}}\right)$. The same subjects were tested in our earlier study [21] using dichoptic temporally sparse multifocal stimuli [30-32], and so we also contrast those results with those obtained with the present two methods. The temporally sparse multifocal stimuli produced sensitivities and specificities for both patient groups around $95 \%$.

\section{Methods}

Subjects and recording

A summary of all subject data is presented in Table 1A. The Normal study group contained 27, the MS study group contained 50 subjects; 26 of the MS patients had experienced ON, 21 of them unilaterally. We will refer to the $M S$ patients with a history of $O N$ as the $M S_{O N}$ patients, and those without a history of $O N$ as the $M S_{N O N}$ patients. MS was diagnosed according to the latest criteria [33]. The subjects' medical history, including the number of MS attacks, MRI, CSF and other relevant details were collected from their neurologist Dr. G. Danta. All MS subjects were classified as relapsing remitting (RR) [33]. Note that both patient groups had very similar durations of disease and a similar number of clinical attacks (Table 1). All patients were diagnosed by the same neurologist, who observed each ON episode.

VEPs were recorded using gold cup electrodes $($ diameter $=8 \mathrm{~mm})$ placed on the scalp with the conductive paste EEG Ten20 (D.O.Weaver and Co, Aurora, LO). Electrodes were attached $3 \mathrm{~cm}$ above and $4 \mathrm{~cm}$ below the inion [34]. An earth electrode was attached to the right ear lobe. The research followed the tenets of the Declaration of Helsinki, under the Australian National University's Human Experimentation Ethics Committee protocol M9901. Informed written consent was obtained from all subjects after the nature and possible consequences of the study were explained to them. 
Table 1 (A) Subject data and (B) four best response components per eye

\begin{tabular}{|c|c|c|c|c|c|c|c|c|}
\hline Study group & $N$ & Age \pm SE $(y r)$ & $\operatorname{Sex}(M / F)$ & Duration of MS (yr) & No. of attacks & ON & CSF & MS type \\
\hline \multicolumn{9}{|c|}{ (A) Subject data } \\
\hline $\mathrm{MS}_{\mathrm{NON}}$ & 24 & $43 \pm 15.2$ & $6 / 18$ & $8.65 \pm 6.6$ & $9.49 \pm 4.8$ & $\mathrm{NO}$ & 7 & RR \\
\hline $\mathrm{MS}_{\mathrm{ON}}$ & 26 & $42 \pm 16.3$ & $2 / 24$ & $8.12 \pm 7.6$ & $10.1 \pm 5.6$ & YES & 9 & RR \\
\hline Normals & 27 & $43.1 \pm 12.1$ & $12 / 15$ & NA & NA & NA & NA & NA \\
\hline \multicolumn{9}{|c|}{ (B) Four best response components per eye } \\
\hline Study group & Total no. of eyes & $>95 \%$ & $>93 \%$ & $>90 \%$ & & & & \\
\hline $\mathrm{MS}_{\mathrm{NON}}$ & 48 & 38 & 48 & 0 & & & & \\
\hline $\mathrm{MS}_{\mathrm{ON}}$ & 52 & 26 & 38 & 52 & & & & \\
\hline Normals & 54 & 46 & 54 & 0 & & & & \\
\hline
\end{tabular}

(A) Subject data. The two columns at the left show the three study groups $\left(\mathrm{MS}_{\mathrm{NON}}, \mathrm{MS}_{\mathrm{ON}}\right.$ and Normals) and the number of subjects $(N)$ who participated in the experiments. MS patients had suffered from the disease on average 8.65 years (see Duration of $M S$ ), and during this time they had approximately 9.49 clinical attacks (see No. of attacks). Twenty six subjects suffered from Optic Neuritis and for 9 of them a CSF oligo-banding test was positive (CSF). MS type was RR for all patients. (B) summarizes the number of significant response components. The Total no of eyes indicates the number of eyes used for our recordings. The three right most columns ( $>95 \%,>93 \%$ and $>90 \%$ ) indicate the number of eyes having 4 or more components at the level of significance for that column

Stimuli

\section{FDT perimeter}

Contrast threshold testing for each of the 17 target locations in the stimulus display (Fig. 1) was performed using, the standard C20 program (FDT full threshold C-20 test, Humphrey, San Leandro, CA) of a FDT perimeter. The FDT stimuli consisted of low-spatial frequency $(0.25 \mathrm{cpd})$ sinusoidal gratings that underwent rapid $(25 \mathrm{~Hz})$ counterphase flicker. Both eyes were tested. Thresholds were expressed as decibel contrast $\left(\mathrm{dB}=20 \log _{10}(\right.$ contrast $\left.)\right)$.

\begin{tabular}{|l|r|r|r|}
\hline 2 & 3 & 4 & 5 \\
\hline 6 & 7 & 8 & 9 \\
\hline 10 & 11 & 12 & 13 \\
\hline 14 & 15 & 16 & 17 \\
\hline
\end{tabular}

Fig. 1 Illustration of the FDT perimeter stimulus regions. Contrast thresholds for spatial frequency doubling illusion stimuli were more determined at each of the 17 regions. The stimuli comprise four regions per quadrant, each $10^{\circ}$ square, and a central $10^{\circ}$ diameter circular region. When active each region displayed a $0.25 \mathrm{cpd}$ grating contrast reversing at $25 \mathrm{~Hz}$
Multifocal frequency doubling visual evoked potentials

For VEP recording, the dichoptic FD visual stimuli were presented on a model CCID 7551 monitor (Barco, Kortrjk, Belgium). A program running on a Vista graphics board (Truevision, Shadeland Station, IN) controlled the stimulus display. Software for data acquisition, analysis and display was written in Matlab (Matlab; The MathWorks, Natick, MA).

Subjects viewed the monitor at $30 \mathrm{~cm}$ providing the angular sub tense of the stimuli illustrated in Fig. 2. The face of the monitor was divided into eight different visual field regions, in which simultaneously presented stimuli were presented. A red fixation spot $\left(0.75^{\circ}\right.$ square $)$ was presented at the screen's centre. Each region contained an achromatic sinusoidal grating at $95 \%$ contrast. All gratings had horizontal stripes. The spatial frequencies of inner regions were $0.4 \mathrm{cpd}$, and the outer regions $0.2 \mathrm{cpd}$. To produce dichoptic stimulation, we used a single monitor and interleaved the images for the left and right eyes on alternate video frames. This was achieved by means of a liquid crystal stereoscopic modulator (or shutter) (Tektronix, Inc., Beaverton, OR, USA) [31, 32]. The shutter and spectacles brought the mean luminance of the display to $7.6 \mathrm{~cd} / \mathrm{m}^{2}$. The noninterlaced refresh rate of the monitor was 


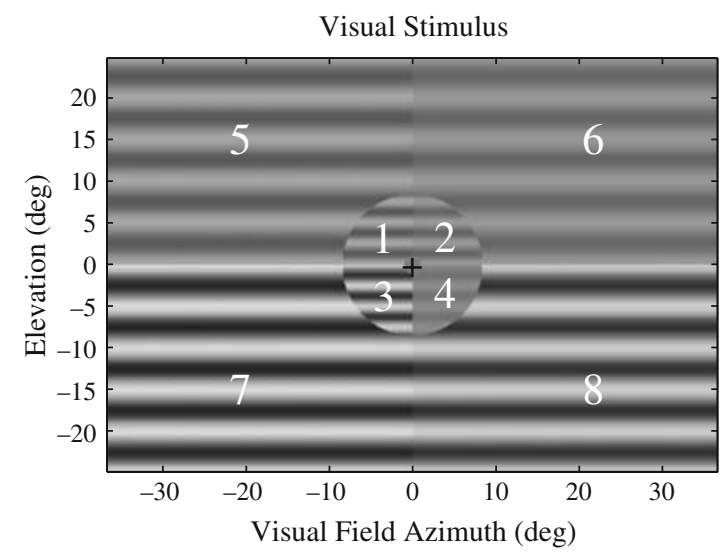

Fig. 2 Illustration of the VEP stimulus appearance. The stimulus screen was divided into 8 concurrently stimulated regions per eye. The central regions were labeled 1-4 and even numbered regions refer to the right visual field. Each region is presented here with a different contrast to make the regions apparent. In practice the regions were concurrently modulated with asynchronous sinusoidal temporal functions, so the figure is a plausible single frame from the total stimulus sequence

129.2 Hz, producing a refresh rate of $64.6 \mathrm{~Hz}$ for each eye following the shutter.

The contrast of each of the resultant 16 grating stimuli ( 8 per eye) was modulated sinusoidally with temporal frequencies ranging from $15.45 \mathrm{~Hz}$ to $21.51 \mathrm{~Hz}$, the left eye receiving the lowest eight frequencies. The spatial and temporal frequencies of the stimuli have previously been shown to produce the FD illusion at the same test locations [35]. Each stimulus sequence lasted $47.5 \mathrm{~s}$ providing a frequency resolution of $\Delta F=0.021 \mathrm{~Hz}$. Four repeats of each testing condition were obtained during the experiment. A fast Fourier transform (FFT) extracted the response components. The recording duration was selected to be the same as for the multifocal case and the trials were done on the same days for each subject to permit comparison of both methods.

In order to employ FFT signal extraction, we needed to create an orthogonal design. Two sinusoids with differing number of complete cycles in the same interval are orthogonal. In this case all 16 frequencies had and integer number of cycles. This insures that each frequency corresponds exactly to a spectral line, and so there is no leakage or other similar effects. Our stimulus frequencies: $f_{1}, f_{2}, \ldots f_{16}$, contained
$\mathbf{N}=3072$ video frames per eye, the actual stimulus frequencies being $\Delta F \times \mathbf{N}$. Because we were interested in the second harmonics, we insured that none of the 240 regional interaction frequencies $\left(f_{i}+f_{j}, i \neq j\right)$ should equal any of the $16 \mathrm{~s}$ harmonic frequencies. Such stimuli are said to be incommensurate $[29,36]$. We also calculated the frequency difference between regions, like regions in the two eyes. They were similar so it was part of the design to try to reduce binocular suppression by making the two eyes as different as possible.

Figure 3 demonstrates graphically the output of the data acquisition program and the initial analysis. Figure 3a (a sample from a Normal subject) and $3 \mathrm{~d}$ (a sample from a $\mathrm{MS}_{\mathrm{NON}}$ subject) show the amplitude spectrum highlighting the fundamental, second and fourth harmonics. Note that the fundamentals are expected to have 0 amplitude as the grating stimuli have almost equal areas of positive and negative contrasts. Figure $3 \mathrm{~b}$, c show the second harmonics numbered 1 to 8 and some other frequencies in the complex plane in an Argand diagram for the left and right eye respectively. In the Argand diagram, frequencies are shown as vectors for which the length from the origin represents signal amplitude and the orientation shows the phase lag. The noise frequency coefficients and regional interaction frequencies (dots) lie mainly inside the circle representing 95\% significance level.

We used a previously described method to determine the significance of the second harmonic responses [29]. The actual significance of a frequency component is related to its amplitude, which is modulus of the Fourier transform coefficient at that frequency. We chose a target inclusion criterion of at least four significant $(P<0.05)$ components per eye for each subject. Twenty three normal subjects met the criterion while the remaining four had four or more significant components at a confidence limit of $93 \%$. At least four significant components per eye were found in $19 \mathrm{MS}_{\mathrm{NON}}$ and $13 \mathrm{MS}_{\mathrm{ON}}$ patients. The remaining patients had four or more responses significant at between $90 \%$ and $93 \%$ confidence. Since the lower amplitudes could be due to damage caused by MS [21], we accepted these subjects. Overall all subjects had at least 


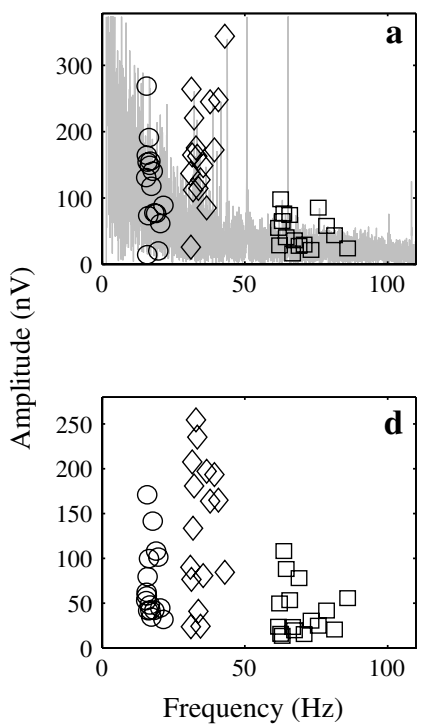

Fig. 3 An example of VEP regional responses. (a) and (d) represent amplitude spectra of exemplary Normal and $\mathrm{MS}_{\mathrm{NON}}$ subjects respectively. The various symbols mark the peaks of potential response components: $(\bigcirc)$ the 16 fundamentals $\left(f_{j}\right),(\diamond)$ second $\left(2 f_{j}\right)$ and $(\square)$ fourth harmonics $\left(4 f_{j}\right)$. (b), (c), (d), (e) and (f) are Argand diagrams showing the responses in the complex plane. (b) and (e) show Argand diagrams for the left eye, panels (e) and (f) give right eye responses. The four segments of each trajectory show the gain and phase for each of four trial

four responses per eye significant at the $90 \%$ level (Table 1B). Note that the values in the cells of the Table 1B are not cumulative, but differential. In the related multifocal ERG study [29] we changed the number of averaged runs until subjects met a criterion number of significant regions. We did four repeats to make a more direct comparison with the sparse multifocal method studied in the same subjects [21].

Note that between subjects VEP phases are relatively similar (e.g. the region 8 vector generally being near the centre of the cluster) but rotated because at these frequencies small differences in delays translate into large phase shifts. Thus, we commonly examined the relative phase, calculated with respect to a reference phase. These relative phases have proved valuable in glaucoma diagnosis using simular stimuli [29]. The reference phase was that of region 8 in each eye (cf. Fig. 2), which on average provided
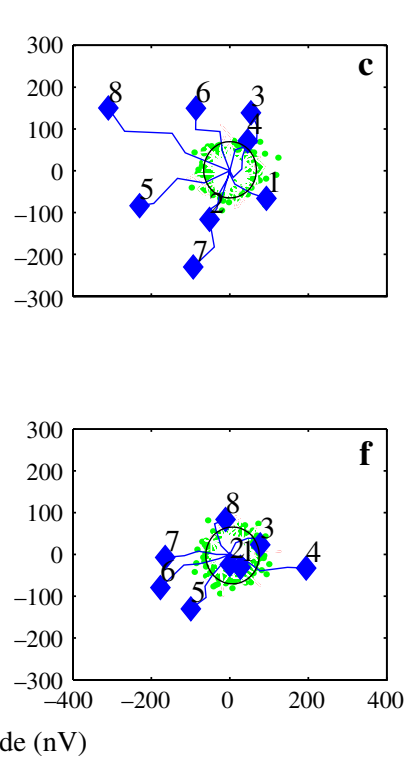

responses $\left(2 f_{j}\right)$, with overall gain scaled by $1 / 4$, so that each trajectory is the vector mean, and its distance from the origin denotes the mean response amplitude. Noise frequencies have random phases and therefore make random walks around the origin. The dashed circle is the $95 \%$ confidence limit computed from the resulting distribution of the noise frequencies. Vectors escaping from the dashed circle with increasing trial number are therefore significantly different from zero. Regional interaction frequencies $f_{i}+f_{j}$ are shown as small dots

the most reliable signal across subjects (Fig. 4a). To construct the relative phases for a given subject, we subtracted the phase of region 8 from each response. Thus, for each eye, the phase of region 8 was thus brought to $0^{\circ}$ while the phase lags and the leads of the other regional responses relative to that of region 8 were preserved.

\section{Data analysis}

\section{FDT and VEP responses}

To compare the 17 FDT thresholds per eye with the 8 VEP regional amplitudes per eye it was necessary to equate the data for stimulus area and position. The overlapping areas of the VEP regions were almost twice those of the FDT regions, therefore we averaged the $\mathrm{dB}$ thresholds of the FDT quadrant regions multiplying each by a 


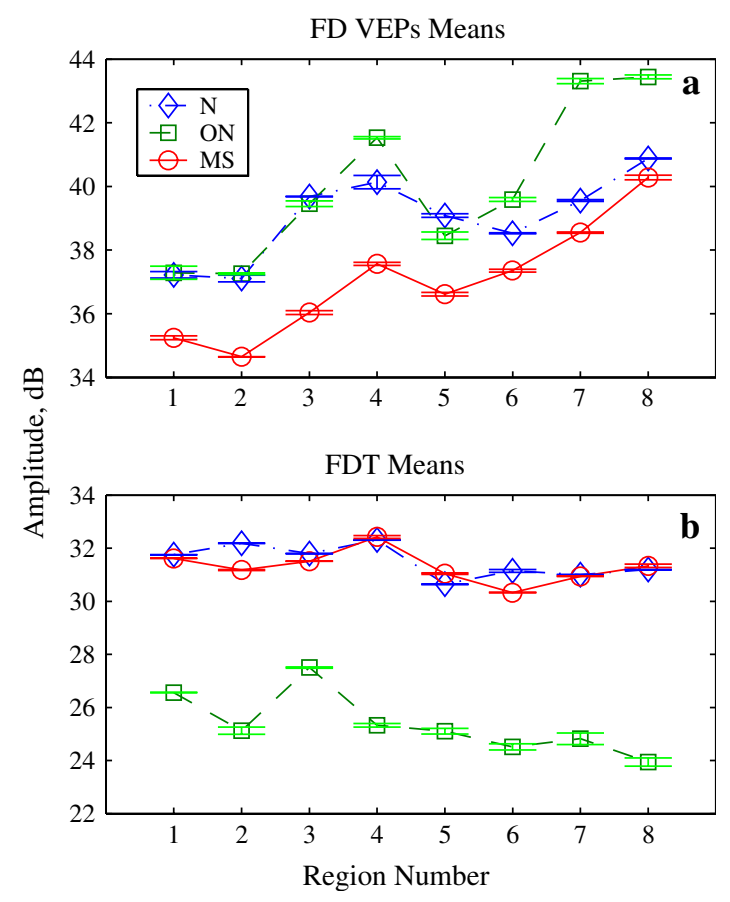

Fig. 4 (a) Regional mfVEP second harmonic responses averaged across subjects and eyes. Separate averages are given for the Normal subjects $(\mathrm{N})$, and the $\mathrm{MS}_{\mathrm{ON}}$ and $\mathrm{MS}_{\mathrm{NON}}$ patients. Error bars are SE. The amplitudes of region 8 were largest on average. Panel (b) represents summarized FDT responses for the same subjects groups

weight $t h_{i}$ in proportion to the overlapping area (Eq. 1).

$R_{\mathrm{FDT}}=\frac{\sum_{i=1}^{4}\left(r_{i} \times \mathrm{th}_{i}\right)}{\sum_{i=1}^{4}\left(\mathrm{th}_{i}\right)}$

where $\mathrm{th}_{i}$ (in $\mathrm{dB}$ ) is the threshold of the FDT test in the $\mathrm{th}_{i}$ region; $r_{i}$ is the weight for $\mathrm{th}_{i}$ and $R_{\text {FDT }}$ is the FDT threshold, transformed into the VEP stimulus dimension. The VEP multifocal responses (in nano volts) were transformed to decibels (Eq. 2). The log transformation had the additional beneficial effect of stabilizing the variance in the VEP values thus permitting additive regression models to be fit to those responses [31].

$\mathrm{dB}_{\mathrm{VEP}}=20 \log _{10}\left(n V_{\mathrm{VEP}}\right)$
Multiple linear regression

Multiple regression was used to quantify various independent effects from the averaged complex Fourier coefficients [29] of the VEP and the thresholds of the FDT. The regression analysis quantified the differences between the Normal and the ON/MS study groups for the FDT and VEP data. In all cases the effect of subject was fit as a nuisance variable. More details of these methods is given elsewhere [31, 32].

\section{Discriminant analysis}

We employed discriminant analysis [37] to construct classifiers to distinguish Normal subjects from the $\mathrm{MS}_{\mathrm{ON}}$ patients. We compared quadratic discriminant analysis (QDA) and linear discriminant analysis (LDA) [21, 29]. QDA models had the capacity to quantify between eye interactions. Diagnostic power was assessed using receiver operator characteristic (ROC) plots [38] constructed from the resulting classifier models. Standard errors for the ROC plots were estimated by a standard bootstrap method where many different data sets were used as a partial validation [21].

Both unsorted and sorted FDT and VEP parameters were included in our classifiers. The sorted measures were reminiscent of conventional perimetric practice where the worst $\mathrm{N}$ regional results are often thought to be diagnostic [39]. The sorting procedure means that regional positional information is lost, however, thus precluding recognition of a particular region or set of regions (superior, central, and peripheral) as being highly diagnostic. We therefore also analysed unsorted responses to search for such possibilities. Scaled regional amplitudes (amplitudes, divided by the geometric mean of the Normal subjects FDT and VEP responses (in $\mathrm{dB}$ )), and also amplitude differences (i.e. differences between the regional amplitudes and their geometric mean across the Normal subjects for the FDT and decibel VEP responses) were considered as well. We examined the minimum, median and maximum values (computed across the regions of 
each eye) of the above mentioned measures obtained for each subject.

\section{Results}

General findings

Figure 4 summarizes the averaged VEPs regional amplitudes and FDT thresholds for Normal (27 subjects), $\mathrm{MS}_{\mathrm{ON}}$ (26 patients) and $\mathrm{MS}_{\mathrm{NON}}$ (24 patients) study groups. Thus smaller $d B$ values indicate lower sensitivity for both FDT and VEP data.

The main effects determining the VEP responses were characterized by multiple regression models. As mentioned in the Methods, good stability of variance was achieved by converting the VEP amplitude into decibels dB (Eq. 2). The transformation to decibels also meant we could also fit the additive models for multiplicative effects such as slightly higher or lower average gains for each subject, a common feature of the data. Table 2A, B summarizes the regression results comparing Normal subjects versus $\mathrm{MS}_{\mathrm{ON}}$ patients and Normal subjects versus $\mathrm{MS}_{\mathrm{NON}}$ patients.
The regression model parameterization set the responses from Normal subjects to be the reference condition. Thus the $t$-value in row one (column labelled ' $t$ ") determines the significance (column labelled " $p$ ") of the mean response amplitude of normal subjects, while the $t$-values in the other rows indicate the significance of the differences between the reference condition (Normals) and the other fitted factors. The values in the Anti-dB column are the inverse of Eq. 2 operated on the fitted $\mathrm{dB}$ valued contents of the Coefficient column. Thus, anti-dB is used in the sense of anti-log and indicates that the values in that column are the real-world values. Hence the value in row one, $89.36 \mathrm{nV}$, is the mean response amplitude for Normal subjects independent of the other fitted factors. The values in the other rows indicate the multiplicative effects of each fitted factor relative the reference condition (Normal).

Thus, the mean effect of being MS patient with no history of ON (Table 2A) is a suppression of response amplitudes in the inner four regions $\left(\mathrm{MS}_{\mathrm{ON}} \times\right.$ inner $)$ by 0.76 times $(-2.32 \mathrm{~dB} \pm 0.63 \mathrm{SE})$ compared to normal subjects, yielding $89.36 \times 0.76=67.91 \mathrm{nV}$. The $-95 \%$ CL and $+95 \%$ CL provide the

Table 2 A and B summarize multivariate regression results for the amplitudes obtained from the Normal versus $\mathrm{MS}_{\mathrm{NON}}$ and $\mathrm{MS}_{\mathrm{ON}}$ subjects

\begin{tabular}{|c|c|c|c|c|c|c|c|}
\hline Condition & Coefficient $(\mathrm{dB})$ & $\mathrm{SE}(\mathrm{dB})$ & $t$ & $P$ & Anti-dB & $-95 \% \mathrm{CL}$ & $+95 \% \mathrm{CL}$ \\
\hline \multicolumn{8}{|c|}{ A. Normals versus $M S_{N O N} \cdot m f V E P$} \\
\hline Ref $=$ Normals & 39.02 & 0.33 & 116.1 & $0.0001<$ & $89.4 \mathrm{nV}$ & 82.8 & 96.1 \\
\hline $\mathrm{MS}_{\mathrm{NON}}$ & -0.11 & 1.00 & -0.11 & 0.6750 & $0.98 \times$ & 0.78 & 1.23 \\
\hline $\mathrm{MS}_{\mathrm{NON}} \times$ inner & -2.32 & 0.63 & -3.64 & 0.0003 & $0.76 \times$ & 0.66 & 0.88 \\
\hline $\mathrm{MS}_{\mathrm{NON}} \times$ age $>40$ & -3.36 & 0.79 & -4.21 & $0.0001<$ & $0.67 \times$ & 0.56 & 0.81 \\
\hline $\mathrm{MS}_{\mathrm{NON}} \times$ male & 2.02 & 1.03 & 1.95 & 0.0544 & $1.26 \times$ & 0.99 & 1.59 \\
\hline \multicolumn{8}{|c|}{ B. Normals versus $M S_{O N} \cdot m f V E P$} \\
\hline Ref $=$ Normals & 39.02 & 0.33 & 119.1 & $0.0001<$ & $89.4 \mathrm{nV}$ & 83.0 & 96.2 \\
\hline $\mathrm{MS}_{\mathrm{ON}}$ & 0.64 & 1.08 & 0.59 & 0.5690 & $1.07 \times$ & 0.84 & 1.37 \\
\hline $\mathrm{MS}_{\mathrm{ON}} \times$ inner & -2.32 & 0.78 & -2.97 & 0.0033 & $0.76 \times$ & 0.64 & 0.91 \\
\hline $\mathrm{MS}_{\mathrm{ON}} \times$ age $>40$ & 2.75 & 0.85 & 3.20 & 0.0015 & $1.37 \times$ & 1.13 & 1.66 \\
\hline $\mathrm{MS}_{\mathrm{ON}} \times$ male & -0.27 & 0.97 & -0.27 & 0.7951 & $0.96 \times$ & 0.77 & 1.21 \\
\hline
\end{tabular}

The VEP amplitudes were transformed into decibels. The data for Normal subjects is set as the reference condition. This design meant multiplicative factors could be fitted and the values in rows $2-5$ of the columns labeled $t$ and $P$ indicate the significance of the decibel difference for each Factor from the mean amplitude for Normal subjects. The decibel regression coefficients (Coefficient), column 2, are the simultaneously fitted values for the Factors described by the left-most column (Condition). The column labeled SE $(d B)$ gives the standard errors in the decibel-valued coefficients. The Anti- $d B$ column indicates the real-world values corresponding to the fitted $\mathrm{dB}$ regression coefficients. For the reference condition, row 1 , the real-value is the mean amplitude for Normal subjects, $89.4 \mathrm{nV}\left(=10^{(39.02 / 20)}\right.$, see Eq. 2$)$. The other rows of the Anti-dB column thus indicate multiplicative scaling values for the other factors corresponding to the fitted dB valued gains. $-95 \% \mathrm{CI}$ and $+95 \% \mathrm{CI}$ indicate confidence intervals in the Anti-dB values 
skewed confidence intervals (due to the initial $\log$ transformation) for each Anti-dB value. We found no significant effect of gender in either the responses of $\mathrm{MS}_{\mathrm{NON}}$ and $\mathrm{MS}_{\mathrm{ON}}$ patients; however a significant decrease of responses $(-3.36 \mathrm{~dB} \pm 0.79 \mathrm{SE})$ was found in $\mathrm{MS}_{\mathrm{NON}}$ patients, older than 40 years. Interestingly, the responses of $\mathrm{MS}_{\mathrm{ON}}$ patients in the same age group increased by $2.75 \mathrm{~dB} \pm 0.85 \mathrm{SE}$ (Table 2B) compared to Normal subjects. The independent effect for the inner four stimulus regions was, however, the same as in $\mathrm{MS}_{\mathrm{NON}}$ patients, a suppression of 0.76 times. The variance accounted for the regression models was $r^{2}=0.62$ for the $\mathrm{MS}_{\mathrm{NON}}$ study group, and 0.64 for the $\mathrm{MS}_{O N}$ patients.

We summarized the FDT threshold effects with similar multiple regression models. Table $3 \mathrm{~A}$ shows the fitted effects of the responses obtained from the Normal and $\mathrm{MS}_{\mathrm{NON}}$ study groups. Table $3 \mathrm{~B}$ indicates the regression results for the FDT thresholds of the Normal and $\mathrm{MS}_{\mathrm{ON}}$ subjects. The table format is the same as for Table 2A, B. The reference condition $(31.51 \mathrm{~dB} \pm 0.24 \mathrm{SE})$ is the mean FDT threshold obtained from Normal subjects. Notice that the Anti-dB value for normal subjects, 37.59 , is their average contrast sensitivity, i.e. the inverse of the average threshold contrast of $2.65 \%$. The values in rows 2 to 5 of the Anti-dB column refer to changes in contrast sensitivity, and so are conceptually similar to the corresponding values in Table 2. There was a significant contrast sensitivity increase in all $\mathrm{MS}_{\mathrm{ON}}$ patients relative to normal subjects of $3.13 \mathrm{~dB} \pm 0.80 \mathrm{SE}(P<0.05)$. Threshold sensitivity significantly decreased in $\mathrm{MS}_{\mathrm{ON}}$ males and also in patients older than 40 years $(-3.52 \mathrm{~dB} \pm 0.63 \mathrm{SE})$. The variance accounted for was $r^{2}=0.67$ for the $\mathrm{MS}_{\mathrm{ON}}$ patients, and 0.74 for the $\mathrm{MS}_{\mathrm{ON}}$ patients.

Note that applying the criteria of $93 \%$ to all subjects (Methods) did not make any significant difference to the above mentioned findings.

Discriminant analysis

LDA and QDA (Methods) were performed on the regional FDT $\left(R_{\mathrm{FDT}}\right.$, Eq. 1$)$ and VEP amplitudes (in $\mathrm{dB}$ ) and/or relative VEP phases. These methods attempt to form the optimum classifier rules for discriminating subject groups based upon linear combinations of test variables (LDA), or quadratic interactions between test variables (QDA). Since data for both left and right eyes was entered QDA had the capacity to exploit between eye interactions, if any, to improve diagnostic accuracy. We examined the performance of parameters, obtained from both eyes assessing the sorted and unsorted minimum, median and maximum (across the visual field

Table 3 A and B summarize multivariate regression results for the FDT amplitudes in decibels of the Normal versus $\mathrm{MS}_{\mathrm{NON}}$ and $\mathrm{MS}_{\mathrm{ON}}$ patients

\begin{tabular}{|c|c|c|c|c|c|c|c|}
\hline Condition & Coefficient (dB) & $\mathrm{SE}(\mathrm{dB})$ & $t$ & $P$ & Anti-dB & $-95 \% \mathrm{CL}$ & $+95 \% \mathrm{CL}$ \\
\hline \multicolumn{8}{|c|}{ (A) Normals versus $M S_{N O N} \cdot F D T$} \\
\hline Ref $=$ Normals & 31.50 & 0.17 & 179.8 & $0.0001<$ & 37.59 & 36.13 & 39.11 \\
\hline $\mathrm{MS}_{\mathrm{NON}}$ & -0.39 & 0.52 & -0.75 & 0.4669 & $0.95 \times$ & 0.84 & 1.07 \\
\hline $\mathrm{MS}_{\mathrm{NON}} \times$ inner & 0.76 & 0.33 & 2.31 & 0.0222 & $1.09 \times$ & 1.01 & 1.17 \\
\hline $\mathrm{MS}_{\mathrm{NON}} \times$ age $>40$ & -0.62 & 0.41 & -1.51 & 0.1378 & $0.93 \times$ & 0.84 & 1.02 \\
\hline $\mathrm{MS}_{\mathrm{NON}} \times$ male & 0.31 & 0.54 & 0.56 & 0.5890 & $1.03 \times$ & 0.91 & 1.17 \\
\hline \multicolumn{8}{|c|}{ B. Normals versus $M S_{O N} \cdot F D T$} \\
\hline Ref $=$ Normals & 31.51 & 0.24 & 129.9 & $0.0001<$ & 37.59 & 35.59 & 39.71 \\
\hline $\mathrm{MS}_{\mathrm{ON}}$ & 3.13 & 0.80 & 3.92 & $0.0001<$ & $1.43 \times$ & 1.31 & 1.57 \\
\hline $\mathrm{MS}_{\mathrm{ON}} \times$ inner & 1.53 & 0.57 & 2.65 & 0.0088 & $1.19 \times$ & 1.12 & 1.27 \\
\hline $\mathrm{MS}_{\mathrm{ON}} \times$ age $>40$ & -3.52 & 0.63 & -5.55 & $0.0001<$ & $0.66 \times$ & 0.62 & 0.72 \\
\hline $\mathrm{MS}_{\mathrm{ON}} \times$ male & -9.28 & 0.72 & -12.82 & $0.0001<$ & $0.34 \times$ & 0.32 & 0.37 \\
\hline
\end{tabular}

The regression model design and the table format are similar to that for Table 2. The reference value $($ Ref $=$ Normals) corresponds to the mean FDT threshold of the Normal subjects, i.e. $31.5 \mathrm{~dB}$. (A) shows the fitted values for the Normal study group versus $\mathrm{MS}_{\mathrm{NON}}$, while (B) presents the fitted values for the FDT thresholds of the Normal versus MS $\mathrm{ON}_{\mathrm{N}}$ study groups 
FDT Thresholds (minimums)

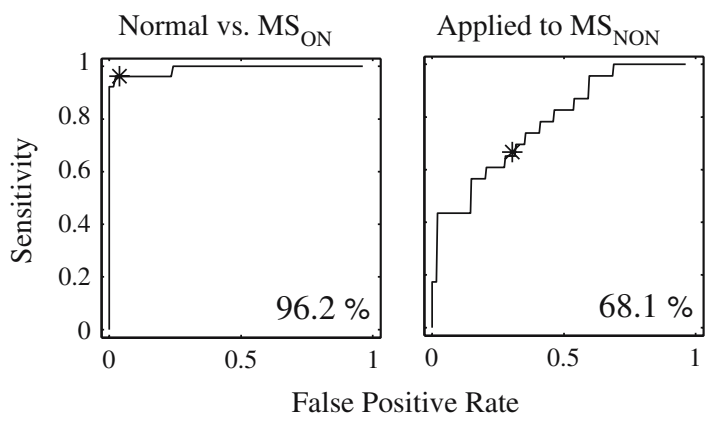

Fig. 5 ROC plots comparing sensitivities and specificities FDT minimum thresholds obtained from each eye. The left panel shows the ROCs for LDA classifier models constructed for the Normal subjects versus $\mathrm{MS}_{\mathrm{ON}}$ patients. The right panel shows the effect of applying that classification model to the $\mathrm{MS}_{\mathrm{NON}}$ patients. The abscissa shows the false positive rates (1-specificity = probability of mis-diagnosing a normal subject), ordinate indicates sensitivities of the model. The '*' symbol represents the point where the highest simultaneous sensitivity and specificity occurs. The mean of these values is shown as a percentage at the bottom right corner of each panel and is sometimes referred to as the accuracy

regions) values of the relative VEP phases, FDT and VEP amplitudes. We first constructed classifiers for the comparison normal subjects versus $M S_{O N}$ patients on the assumption that $\mathrm{MS}_{\mathrm{ON}}$ patients would show damage due to MS in visual test. We then applied those classifiers to the comparison normal subjects versus $M S_{N O N} p a-$ tients, so that using the same classifier we could see how well the rule based on $\mathrm{MS}_{\mathrm{ON}}$ subjects applied to distinguishing the $\mathrm{MS}_{\mathrm{NON}}$ group. Although many models were examined those of most interest are summarized below.

\section{Diagnosis with FDT thresholds}

We were interested to know how the FDT thresholds performed on their own. The performance of the left and right minimum (worst) thresholds obtained from both eyes was high: the model performed at a sensitivity of $96.29 \%$ and a specificity of $96.15 \%$ for LDA. QDA sensitivities and specificities were virtually the same as for LDA indicating that there was no diagnostic value for an interaction between eyes. This was typical for all classifiers and so QDA results will not be mentioned henceforth. The median values of the FDT thresholds performed at a lower sensitivity of $80.76 \%$ and a specificity of $81.48 \%$ for LDA.

The performance of the model containing the FDT minimum thresholds is shown in the Fig. 5. The left panel displays the ROC plots (Methods) for the Normal versus $\mathrm{MS}_{\mathrm{ON}}$ patients. The right panel shows the model that applied to the data of $\mathrm{MS}_{\mathrm{NON}}$ patients. The '*' symbol in each panel indicates the point of the simultaneously largest sensitivities and specificities, the mean of these values is sometimes called the accuracy. The \% value of the resulting accuracies is at the lower right quadrant of each panel. As illustrated in the figure, minimum FDT amplitude values had accuracies of $96.2 \%$ for $\mathrm{MS}_{\mathrm{ON}}$ patients and $68.1 \%$ for $\mathrm{MS}_{\mathrm{NON}}$.

\section{VEP regional amplitudes}

We also examined discriminant models based upon VEP responses. The minimum VEP amplitudes obtained from both eyes performed at a sensitivity of $48.1 \%$ and at a specificity of $50 \%$. The median amplitudes were similar at $57.6 \%$ sensitivity at a specificity of $55.5 \%$. A sensitivity of $61.5 \%$ at specificity of $62.9 \%$ was achieved for the maximum VEP in $\mathrm{MS}_{\mathrm{ON}}$ patients (Fig. 6), while essentially chance performance was given for the $\mathrm{MS}_{\mathrm{NON}}$ group.

\section{Relative VEP phases}

We were also interested to know how the relative phases (Methods) would perform because they

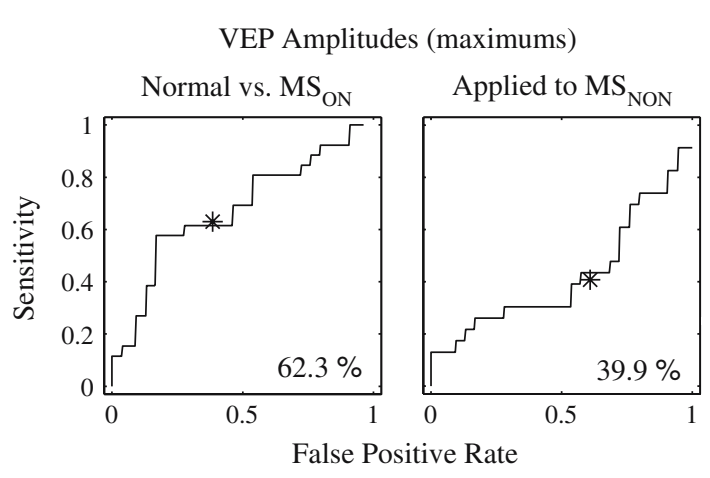

Fig. 6 ROCs for the maximum VEP values recorded from each eye. The figure format is as for Fig. 5 


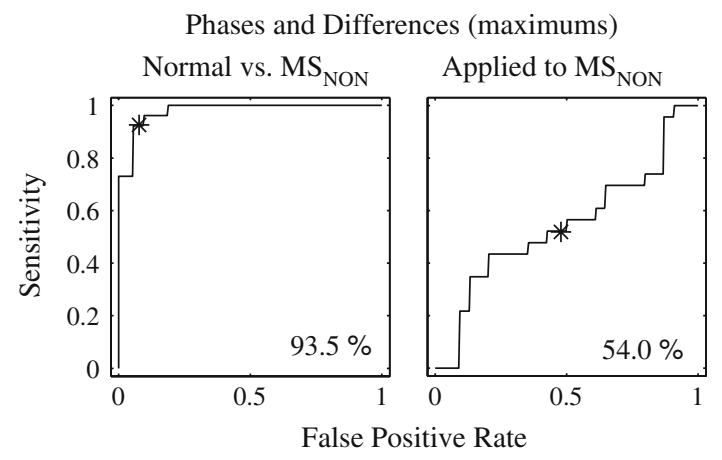

Fig. 7 ROCs for the maximum VEP phase and FDT threshold differences $\left(\mathrm{FDT}_{\mathrm{D}}\right.$ and $\left.\mathrm{VEP}_{\mathrm{D}}\right)$ obtained from both eyes. The figure format is as for Fig. 5

had proved to be diagnostic in glaucoma [29]. The resulting LDA model, based on the maximum values of the relative phases obtained from the Left and Right eyes, from $\mathrm{MS}_{\mathrm{ON}}$ and Normal subjects gave a sensitivity of $65.3 \%$ at a specificity of $80.4 \%$. Only $53 \%$ of $\mathrm{MS}_{\mathrm{NON}}$ patients were classified correctly.

Given the results summarized in Table 3A, B we were interested to know whether the data of the inner regions were able to give higher performance in $\mathrm{MS}_{\mathrm{ON}}$ and $\mathrm{MS}_{\mathrm{NON}}$ patients. The performance of the classifier model containing the minimum values of the FDT inner regions was $82.1 \%$ for $\mathrm{MS}_{\mathrm{ON}}$ and $62 \%$ for $\mathrm{MS}_{\mathrm{NON}}$. It increased to $65 \%$ in $\mathrm{MS}_{\mathrm{NON}}$ patients for the minimum VEP inner regional amplitudes.

\section{Combining FDT and VEP}

We also examined the diagnostic accuracy of models that combined FDT and VEP data. The best performance was achieved for models that included the minimum four measures from each test and eye. In this case sensitivity reached $96.15 \%$ at a specificity of $96.29 \%$ in Normal versus $\mathrm{MS}_{\mathrm{ON}}$ patients. It seems that sensitivity of this model was determined largely by the FDT thresholds. When the same model was applied to $\mathrm{MS}_{\mathrm{NON}}$, its performance dropped to a sensitivity of $65.2 \%$ at a specificity of $66.3 \%$, similar to Fig. 5. We then selected the eight worst FDT and VEP results from the two eyes. Minimum, median and maximum measures gave sensitivities and specificities of $100 \%$ in $\mathrm{MS}_{\mathrm{ON}}$ patients, but accuracies of only $55 \%$ were obtained for $\mathrm{MS}_{\mathrm{NON}}$ patients (Fig. 7).

Figure 8 illustrate the resulting mean and SE of the bootstrapped ROCs for the FDT thresholds, mfVEPs amplitudes, phases and differences. We used 500 bootstrapped data sets per ROC. The SEs is the standard deviations in the mean bootstrapped ROC.

\section{Discussion}

\section{General findings}

The mfVEPs technique is one of the most important parts of this paper. The VEPs were recorded from eight different visual field regions, in which simultaneously presented stimuli were shown. The stimuli for the eight locations and two eyes were modulated with different temporal frequencies. The negative point of this analysis, that both the signal to noise (SNR) ratio and phase of the VEP were influenced by the temporal frequency of the contrast modulation. The problems of variable SNR with frequency and ability to compare between eyes would be minimized by making the stimulus frequencies closer together. That could be done by using multiple regression rather than FFT to extract the responses. As no one else has tried anything like this method before, it was worth a try the simpler form of analysis to feel the prospects.

We found that overall $\mathrm{MS}_{\mathrm{ON}}$ patients actually had greater contrast sensitivities than Normal subjects by $3.13 \mathrm{~dB}(P<0.0001)$ (cf. Table 3$)$. $\mathrm{MS}_{\mathrm{ON}}$ patients, older than 40 years (Table $3 \mathrm{~B}$ ) were, however, less sensitive by $-3.52 \mathrm{~dB} \pm 0.63 \mathrm{SE}$ $(P<0.0001)$. The results for older $\mathrm{MS}_{\mathrm{ON}}$ patients probably occurred because of their longer history of the disease and the larger number of relapses that those patients experienced. The FDT thresholds of the $\mathrm{MS}_{\mathrm{NON}}$ patients differed from Normal subjects in being 1.09 times $(P=0.022)$ more sensitive in their inner four stimulus regions.

Interestingly, the VEPs of $\mathrm{MS}_{\mathrm{ON}}$ patients, older than 40 years of age, were larger than those of Normal subjects by $2.75 \mathrm{~dB} \pm 0.85 \mathrm{SE}$ $(P=0.0015)$ (Tables $2 \mathrm{~B})$ and this was independent 
of any effect of gender. The mfVEP responses obtained for the superior visual field were about $2 \mathrm{~dB}$ smaller, but probably due to electrode position (21), so factor was not shown in Table 2 although it was fitted.

\section{Discriminant analysis}

Fujimoto and Adachi-Usami [18] examined 12 $\mathrm{MS}_{\mathrm{ON}}$ patients using Humphrey achromatic and FDT perimetry. FDT indicated a depression in the extra foveal area. Our findings agree with that study in that, relative to inner regions, the thresholds for outer regions were suppressed by $0.76 \mathrm{~dB}$ in our $\mathrm{MS}_{\mathrm{NON}}$ patients, and $1.53 \mathrm{~dB}$ in our $\mathrm{MS}_{\mathrm{ON}}$ patients (Table 3 ). By contrast in our VEP results it was the inner regions that were suppressed by $-2.32 \mathrm{~dB}$ in both patients groups (Table 2). The study of Wall et al. [19] was designed to determine sensitivity and specificity of conventional automated perimetry and FDT in both optic neuropathy and ON. They found that sensitivities and specificities of FDT and conventional perimetry were similar respectively providing a sensitivity of $81.3 \%$ at a specificity of $76.2 \%$, and $87.5 \%$ at $81.0 \%$. Studies examining molecular markers for MS typically have reported sensitivities obtained at $95 \%$ specificity. Although sensitivities of $92 \%$ have been reported for such markers [40] sensitivities closer to $60-75 \%$ are more common [41, 42]. Earlier reports of the sensitivity of conventional VEPs indicate they are in the range $67 \%$ [43] to $81 \%$ [44]. Thus, the present results (Fig. 7) represent an improvement over molecular markers and standard VEPs, at least for MS patients with a history of ON.

In the present study good performance was observed in $\mathrm{MS}_{\mathrm{ON}}$ patients for both the FDT and mfVEP (accuracies over 90\%) when the results from the worst performing regions of each eye were considered (Figs. 5 and 7). Our somewhat higher accuracies for FDT compared to Walls et al. [19] may be due to possibly more advanced disease in our patients, and or to the action of spatial averaging (Eq. 1). The classification of $\mathrm{MS}_{\mathrm{NON}}$ patients was poor for all classifier models examined. This was expected as Fig. 4 shows that the FD responses in the Normal subjects and $\mathrm{MS}_{\mathrm{NON}}$ patients with no visual symptoms were not significantly different (cf. Tables $2 \mathrm{~B}, 3 \mathrm{C}$ ).

We constructed LDA and QDA variants of every classification model examined. As mentioned above (Methods) we used the criterion of 4 or more VEP amplitudes at the level of significance to determine the inclusion of Normal subjects. Four out of 27 Normal subjects were rejected based on this criterion, but some patients were not rejected since their lower amplitudes could be due to the damage of the optic nerve. Although QDA had the capacity to
Fig. 8 Mean \pm SE of

bootstrapped $(n=500)$

ROC plots for the

minimum FDT

thresholds, maximum

VEP values and the

maximum VEP phase and

FDT threshold

differences. The top row

indicates ROCs for the

normal subjects and

$\mathrm{MS}_{\mathrm{ON}}$ patients; the

bottom row represents the

ROCs for the normal

subjects and $\mathrm{MS}_{\mathrm{NON}}$

patients

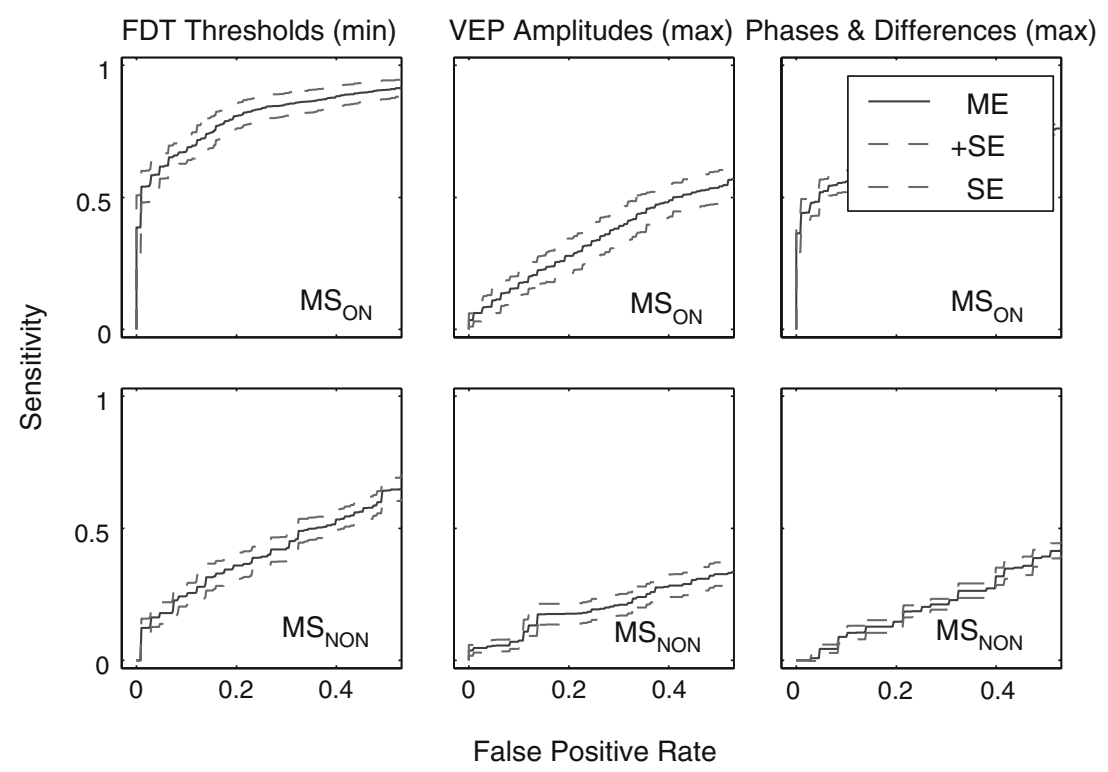


exploit between eye differences no QDA classifier significantly outperformed any LDA classifier examined, suggesting rather uniform damage to the CNS. This was in spite of the fact that 21 of the $26 \mathrm{MS}_{\mathrm{ON}}$ patients had only had unilateral ON. The LDA versus QDA comparison was a sensitive one given that the data for the two eyes was obtained concurrently in every subject.

\section{Temporally sparse stimuli}

The present results are in great contradistinction to those that we reported earlier which were obtained in the same subjects [21]. The stimuli employed in that study were temporally sparse dichoptic mfVEP stimuli. The most temporally sparse stimuli yielded accuracies $>95 \%$ in both $\mathrm{MS}_{\mathrm{ON}}$ and $\mathrm{MS}_{\mathrm{NON}}$ patients. Those data where obtained in the same recording sessions and from the same subjects as the present results. The recording duration for the sparse stimuli, four repeats of a $40 \mathrm{~s}$ stimulus, was actually shorter than present FD mfVEP stimulus, but the number and layout of the stimulus regions was the same. Sparse multifocal stimuli generate responses that are up to 15 times larger than conventional multifocal VEP stimuli [30, 31], providing highly accurate estimates of conduction delays, the measure yielding the high observed accuracies for both patient groups.

\section{Conclusions}

Overall, the measures investigated here for FD stimuli, both threshold and suprathreshold, seem only to have diagnostic value in $\mathrm{MS}_{\mathrm{ON}}$ patients. That being said only RR patients were studied here, it would be interesting to examine primary progressive patients with FD stimuli given that generalized CNS atrophy is characteristic of that disorder [45, 46]. The fact that the temporally sparse multifocal stimuli could diagnose the $\mathrm{MS}_{\mathrm{NON}}$ group well may indicate that it is more tuned to the progressive phase of the disease rather than the inflammatory component as the $\mathrm{MS}_{\mathrm{NON}}$ clearly have had less severe inflammatory disease in their optic nerves [21]. Neither of the FD stimuli used here seem to share this property.

\section{References}

1. Kelly D (1966) Frequency doubling in visual responses. J Opt Soc Am 56(11):1628-1633

2. Kelly D (1981) Nonlinear visual responses to flickering sinusoidal gratings. J Opt Soc Am 71(9):1051-1055

3. Tyler CW (1974) Observations on spatial-frequency doubling. Perception 3:81-86

4. Maddess T, Kulikowski J (1999) Apparent fineness of stationary compound gratings. Vision Res 39:34043416

5. Kulikowski JJ (1975) Apparent fineness of briefly presented gratings: balance between movement and pattern channels. Vision Res 15:673-680

6. Maddess T, Henry G (1992) Nonlinear visual responses and visual deficits in ocular hypertensive and glaucoma subjects. Clin Vis Sci 7:371-383

7. Maddess T, Severt W, Stange G (2001) Comparison of three tests using the frequency doubling illusion to diagnose glaucoma. Clin Exp Ophthalmol 29:359-367

8. Maddess T, Goldberg I, Wine S, Dobinson J, Welsh AH, James AC (1999) Testing for glaucoma with the spatial frequency doubling illusion. Vision Res 39:4258-4273

9. Maddess T (2000) Perspectives on the use of frequency doubling and short wavelength perimetry for the diagnosis of glaucoma. Clin Exp Ophthalmol 28:245247

10. Maddess T, James A, Goldberg I, Wine S, Dobinson J (2000) Comparing a parallel PERG, automated perimetry, and frequency-doubling thresholds. Invest Ophthalmol Vis Sci 41(12):3827-3832

11. Alward WLM (2000) Editorial: frequency doubling perimetry for the detection of glaucomatous field loss. Am J Ophthalmol 129:376-378

12. Cello K, Nelson-Quigg J, Johnson C (2000) Frequency doubling technology perimetry for detection of glaucomatous visual field loss. Am J Ophthalmol 129:314322

13. Chauhan B, Johnson C (1998) Test-retest variability characteristics of frequency doubling perimetry and conventional perimetry in patients with glaucoma and normal controls. Invest Ophthalmol Vis Sci 39:655-658

14. Blumenthal E, Sample P, Zangwill L, Lee A, Kono Y, Weinreb R (2000) Comparison of long term variability for standard and short-wavelength automated perimetry in stable glaucoma patients. Am J Ophthalmol 129:309-313

15. Heijl A, Lindgren A, Lindgren G (1989) Test-retest variability in glaucomatous visual fields. Am J Ophthalmol 108:130-135

16. Artes P, Iwase A, Ohno Y, Kitazawa Y, Chauhan B (2002) Properties of perimetric threshold estimates from full threshold, SITA Standard, and SITA Fast Strategies. Invest Ophthalmol Vis Sci 43:2654-2659

17. Wall M, Johnson CA, Kutzko KE, Nguyen R, Brito C, Keltner JL (1998) Long- and short-term variability of automated perimetry results in patients with optic neuritis and healthy subjects. Arch Ophthalmol 116(1):53-61 
18. Fujimoto N, Adachi-Usami E (2000) Frequency doubling perimetry in resolved optic neuritis. Invest Ophthalmol Vis Sci 41:2558-2560

19. Wall M, Neahring RK, Woodward KR (2002) Sensitivity and specificity of frequency doubling perimetry in neuro-ophthalmic disorders: a comparison with conventional automated perimetry. Invest Ophthalmol Vis Sci 43(4):1277-1283

20. Minagar A, Toledo EG, Alexander JS, Kelley RE (2004) Pathogenesis of brain and spinal cord atrophy in multiple sclerosis. J Neuroimaging 14(3 Suppl):5S-10S

21. Ruseckaite R, Maddess T, Danta G, Lueck C, James A (2005) Sparse multifocal stimuli for the detection of multiple sclerosis. Ann Neurol 57:904-913

22. Compston A, Coles A (2002) Multiple sclerosis. Lancet 359(9313):1221-1231

23. Ferguson B, Matyszak MK, Esiri MM, Perry VH (1997) Axonal damage in acute multiple sclerosis lesions. Brain 120 (Pt 3):393-399

24. Andersson T, Siden A, Persson A (1991) A comparison of clinical and evoked potential (VEP and median nerve SEP) evolution in patients with MS and potentially related conditions. Acta Neurol Scand 84(2):139145

25. Brasil Neto J (1991) Evoked potentials in multiple sclerosis: recent experience at the Locomotor System Diseases Hospital. Arq Neuropsiquiatr 49(2):204-207

26. Sand T, Sjaastad O, Romslo I, Sulg I (1990) Brain-stem auditory evoked potentials in multiple sclerosis: the relation to VEP, SEP and CSF immunoglobulins. J Neurol 237(6):376-378

27. Roder H (1991) VEP in the determination of multiple lesions in the visual system in patients with multiple sclerosis. EEG EMG Z Elektroenzephalogr Elektromyogr Verwandte Geb 22(4):234-238

28. Hood D, Odel J, Zhang X (2000) Tracking the recovery of local optic nerve function after optic neuritis: a multifocal VEP study. Invest Ophthalmol Vis Sci 41(12):4032-4038

29. Maddess T, James A, Goldberg I, Wine S, Dobinson J (2000) A spatial frequency - doubling illusion - based pattern electroretinogram for glaucoma. Invest Ophthalmol Vis Sci 41(12):3818-3826

30. James A (2003) The pattern pulse multifocal visual evoked potential. Invest Opthalmol Vis Sci 44(2):879890

31. James A, Ruseckaite R, Maddess T (2005) Effect of temporal sparseness and dichoptic presentation upon multifocal visual evoked potential. Vis Neurosci 22:4554

32. Maddess T, James A, Bowman EA (2005) Contrast response of temporally sparse and contrast reversing dichoptic multifocal evoked potentials. Visual Neurosci $22: 153-162$
33. McDonald W, Compston A, Edan G, Goodkin D, Hartung H, Lublin F et al (2001) Recommended diagnostic criteria for multiple sclerosis: guidelines from the international panel on the diagnosis of multiple sclerosis. Ann Neurol 50:121-127

34. Klistorner A, Graham S, Grigg J, Billson F (1998) Electrode position and the multi-focal visual-evoked potential: role in objective visual field assessment. Aust N Z J Ophthalmol 26(Suppl.):91-94

35. Bedford S, Maddess T, Rose K, James A (1997) Correlations between observability of the spatial frequency doubled illusion, a multi-region PERG. Aust N Z J Ophthalmol 25:91-93

36. Victor J, Shapley R (1980) A method of nonlinear analysis in the frequency domain. Biophys J 29(3):459483

37. Johnson R, Wichern D (1992) Applied multivariate statistical analysis, 3rd edn. Prentice-Hall, Inc

38. Egan J (1975) Signal detection theory and ROC analysis. London, Academic Press, Inc. Ltd

39. Capriolli J (1991) Automated perimetry in glaucoma. Am J Ophthalmol 111:235-239

40. McMillan SA, McDonnell GV, Douglas JP, Hawkins SA (2000) Evaluation of the clinical utility of cerebrospinal fluid (CSF) indices of inflammatory markers in multiple sclerosis. Acta Neurol Scand 101(4):239 243

41. Chamczuk AJ, Ursell M, O’Connor P, Jackowski G, Moscarello MA (2002) A rapid ELISA-based serum assay for myelin basic protein in multiple sclerosis. J Immunol Methods 262(1-2):21-27

42. Brasher GW, Follender AB, Spiekerman AM (1998) The clinical value of commonly used spinal fluid diagnostic studies in the evaluation of patients with suspected multiple sclerosis. Am J Manag Care 4(8):1119-1121

43. Ravnborg M, Liguori R, Christiansen $\mathrm{P}$, Larsson $\mathrm{H}$, Sorensen P (1992) The diagnostic reliability of magnetically evoked motor potentials in multiple sclerosis. Neurol 42(7):1296-1301

44. van Diemen HA, Lanting P, Koetsier JC, Strijers RL, van Walbeek HK, Polman CH (1992) Evaluation of the visual system in multiple sclerosis: a comparative study of diagnostic tests. Clin Neurol Neurosurg 94(3):191-195

45. De Stefano N, Matthews PM, Filippi M, Agosta F, De Luca M, Bartolozzi ML et al (2003) Evidence of early cortical atrophy in MS: relevance to white matter changes and disability. Neurology 60(7):1157-1162

46. Clanet M, Cassol E, Manelfe C, Berry I (2003) Clinical-MRI correlations in the secondary progressive phase of MS: lessons from the treatment trials. J Neurol Sci 206(2):139-144 